CRÍTICA, Revista Hispanoamericana de Filosofía

Vol. XXXI, No. 93 (diciembre 1999): 49-74

\title{
SEMÁNTICA ASPECTUAL Y PREDICACIÓN SECUNDARIA
}

\author{
VÍctor SÁnchez VALEncia \\ Facultad de Letras \\ Universidad de Groningen
}

\section{Introducción}

En este artículo intentaremos desarrollar una representación semántica que capture el contenido del tipo de oración ejemplificado por las tres oraciones siguientes:

(1) a. María arribó contenta

b. María habló dormida

c. María escribió la carta nerviosa

Antes que nada deseo hacer dos observaciones preliminares acerca de la terminología que va a ser empleada en este artículo. Después describiré su estructura general y su propósito central.

\section{Predicación descriptiva}

La primera observación concierne a las expresiones que vamos a emplear para referirnos a oraciones similares a las presentadas en (1). Diremos que estas oraciones contienen dos expresiones predicativas:

1. En primer lugar el predicado expresado por los verbos arribar, hablar, escribir. 
2. En segundo lugar el predicado adjetival, contenta, dormida, nerviosa, en concordancia con el sujeto.

En la terminología lingüística contemporánea, el predicado verbal es llamado predicado primario mientras que el predicado adjetival recibe el nombre de predicado secundario. En la literatura anglosajona este predicado secundario es usualmente llamado depictive. Para aliviar la monotonía de nuestro metalenguaje, adoptaremos el término descriptivo como la contraparte en castellano al vocablo inglés. Así pues, hablaremos indistintamente de 'oraciones descriptivas' o de 'oraciones de predicación secundaria'. ${ }^{1}$

\section{Modos de acción}

La otra observación concierne al uso de expresiones tomadas del estudio del aspecto. Los términos estado, logro, actividad y realización son usados para hablar de tipos de acción y constituyen una parte del metalenguaje lingüístico contemporáneo. Éstos corresponden, respectivamente, con los términos state, achievement, activity y accomplishment. Según una de las concepciones en voga, diversos tipos de acción pueden ser distinguidos con base en un número finito de propiedades temporales: telicidad, duratividad, dinamismo. Ilustremos esto por medio de los siguientes ejemplos:

1. un estado es un tipo de acción atélico, durativo y estático: estudiar lingüística;

2. una actividad es un tipo de acción atélico, durativo y dinámico: caminar, dormir;

1 La predicación secundaria ha sido estudiada por diversos autores, incluyendo Green 1970, Dowty 1972, Nichols 1978, Luján 1980, Rothstein 1985, Demonte 1988, Napoli 1989, Guemann 1990, Grubig 1992, Larson y Segal 1995, Winkler 1996. 
3. un logro es un tipo de acción télico, puntual y dinámico: encontrar la llave, alcanzar la cima, arribar;

4. una realización es un tipo de acción télico, durativo y dinámico: escribir una carta.

De acuerdo con esta tipología (la) denota un logro, (lb) denota una actividad y (lc) denota una realización. Como se verá más adelante, la semántica de las oraciones descriptivas es sensible a estas distinciones tipológicas.

Otras convenciones terminológicas serán establecidas cuando surja la necesidad de hacerlo así. Este artículo está organizado de la siguiente forma: En la segunda sección bosquejamos el tratamiento de la modificación adverbial propuesta por Davidson. En la tercera parte describiremos la manera en que Dowty analizó la predicación secundaria y la recepción que este análisis ha gozado. La siguiente tarea es la extensión del análisis de Dowty. De hecho, lo que proponemos es la incorporación de este análisis dentro de una semántica aspectual. La ventaja de este paso consiste en la aclaración de ciertos desacuerdos que se han dado en torno a la interpretación más adecuada de las oraciones descriptivas. Como se verá, el punto de vista que adoptamos nos permite concluir que el desacuerdo es más aparente que real. El punto de partida de mi análisis es ajeno a todo tipo de controversia. Doy por hecho que una oración descriptiva expresa una relación temporal entre su predicado primario y su predicado secundario. Concretamente, adopto el punto de vista de que el contenido semántico de esta relación temporal está ligado estrechamente al modo de acción expresado por el predicado primario. Como veremos, el resultado de este tratamiento es que la semántica que ha sido propuesta para la conectiva temporal inglesa when y la semántica para la predicación descriptiva coinciden. 


\section{Eventualidades y adjetivos adverbiales}

El análisis Davidsoniano del lenguaje natural presupone la existencia de un conjunto no vacío de eventualidades. Éstas pueden ser sujetas a procesos de referencia y además pueden servir para la interpretación de variables individuales. Eventualidad es el término técnico introducido en Bach (1981) para cubrir estados, actividades, logros y realizaciones. Frases verbales y adverbiales son, según Davidson, interpretables como predicados de eventualidades. Considérese, por ejemplo, la oración (2)

(2) María arribó rápido

En esta oración el adjetivo rápido carece de concordancia con el sujeto y es tomada como expresión de modificación adverbial. Como acabamos de decir, este tipo de modificación es interpretado por Davidson como predicación sobre eventualidades. De esta forma, una representación Davidsoniana de (2) en la versión modificada propuesta por Parsons (1985) es (3):

(3) $\exists$ e $(\operatorname{arribar}(\mathrm{e}) \wedge$ actor(e, María) $\wedge$ rápido(e) $)$

Según esta interpretación, (2) significa que existe una eventualidad de arribar y que esta eventualidad tiene la propiedad de ser una eventualidad rápida. Éste es el contenido de la fórmula en (3).

Esta representación de los adjetivos adverbiales sugiere una representación análoga de la oraciones descriptivas. Por ejemplo, (la) puede ser asociada con (4):

(4) $\exists$ e $(\operatorname{arribar}(e) \wedge$ actor(e, María) $\wedge$ contenta(María))

Por consiguiente, se considera que (la) expresa la existencia de una eventualidad de arribar, que María es el agente de esta eventualidad y que este agente tiene la propiedad adicional de estar contenta. Éste es el contenido de (4). 
La motivación de este artículo es la constatación de que (4) no alcanza a capturar importantes intuiciones semánticas con respecto a la predicación descriptiva. Ningún hablante del español puede soslayar la fuerte intuición de que el predicado descriptivo modifica al predicado verbal y no sólo al actor de la eventualidad. Esta intuición es expresada en la Real Academia de la Lengua $(1985$, p. 369) al decir que en la predicación descriptiva el adjectivo expresa una cualidad o estado del subjeto expresando al mismo tiempo una modificación adverbial del verbo.

Larson y Segal han explorado existosamente un modo de mejorar (4). Sin embargo, existe una elegante alternativa para obtener la misma optimalización. Esta alternativa será la que desarrollaremos aquí. Hablando en términos generales damos por hecho que

- la relación temporal relevante para la predicación descriptiva existe entre logros, actividades y realizaciones por una parte y estados por la otra;

- esta relación temporal es la inclusión temporal o la coincidencia parcial;

- el tipo de eventualidad del predicado primario está correlacionado con la naturaleza de la relación temporal involucrada.

De especial importancia es la siguiente observación. En las cláusulas temporales construidas con la conectiva when, el tipo de eventualidad de la oración principal induce la misma relación temporal entre la oración principal y la oración subordinada como ocurre en las oraciones descriptivas. Nosotros podemos ver aquí que este hecho liga el análisis de la predicación secundaria que ofrecemos a la teoría propuesta por Dowty (1972). 
Antes de desarrollar los detalles de nuestra propuesta, presentaremos un breve resumen del tratamiento que Dowty le confiere a la predicación descriptiva.

\section{Dowty y la predicación descriptiva}

En su artículo (Dowty 1972), David Dowty se ocupa de la oración en (5) la cual es obviamente similar a las oraciones en (1).

(5) The girl married young

La muchacha se casó joven

Dowty llama adjetivos restringidos temporalmente a los predicados secundarios. En su opinión estos adjectivos actúan semánticamente de una manera similar a como lo hacen las cláusulas de tipo when. De este modo, él ofrece parafrasear (5) como

(6) The girl married when she was young La muchacha se casó cuando era joven

Esta paráfrasis con el auxilio de when muestra que Dowty adopta un marco adverbial para la interpretación de los predicados descriptivos. El adjetivo impone el marco temporal dentro del cual se desenvuelve la eventualidad que corresponde al predicado verbal.

La paráfrasis de Dowty ha sido rechazada (Luján 1982). Luján argumenta que la mayor restricción de esta propuesta es su limitado alcance. De acuerdo con ella, esta paráfrasis no funciona más que para una clase reducida de adjetivos que denotan propiedades temporales. En relación con otros predicados esta paráfrasis está lejos de ser satisfactoria. Por ejemplo, desde su punto de vista, las oraciones (a) que enseguida se muestran no son equivalentes a las oraciones (b) correspondientes:

(7) a. La maestra llegó cansada 
b. La maestra llegó cuando estaba cansada

(8) a. Nació ciega

b. Nació cuando era ciega

Imbuido en el mismo escepticismo está el comentario de Steven Guemann. En su tesis doctoral (Guemann 1990, p. 179), él acusa a Dowty de confundir los predicados descriptivos con los predicados adverbiales. En su opinión, el análisis propuesto por Dowty es inaceptable. Existen, para él, mejores paráfrasis que la que Dowty adoptó. Así pues, mejores interpretaciones de (5) y de (8) son, respectivamente, (9a) y (9b):

(9) a. When the girl married she was young

b. Cuando nació estaba ciega

Esta observación refleja ciertas dudas que el mismo Dowty expresara. Nosotros tocaremos este punto un poco más adelante. Por el momento quiero subrayar que hay un acuerdo general con respecto a la relación temporal entre las eventualidades involucradas en la predicación descriptiva. Las dudas conciernen a la naturaleza de la relación no a su existencia. Éste es un punto de crucial importancia y merece confirmación adicional. Bernhard Grubig notó que la predicación descriptiva está determinada, parcialmente, por la subordinación temporal del predicado descriptivo al predicado primario y por el hecho de que el predicado descriptivo predica el estado de su argumento mientras que la eventualidad asociada con el predicado principal toma lugar. (Cfr. Grubig 1992, p. 178). De igual modo, Violeta Demonte (Demonte 1988, p. 3) toma a (10b) como la paráfrasis que corresponde con (10a).

(10) a. Juan come la carne cruda

b. Juan come la carne mientras está cruda 
Para estos dos ejemplos es preferible una paráfrasis basada en la conectiva temporal mientras. La posición sugiere una preferencia por la interpretación en la que el predicado principal corresponde con una cláusula de when. Estas dos posiciones contrastan con la línea adoptada por Dowty. Esta falta de acuerdo con respecto a la naturaleza exacta de la relación temporal demanda una interpretación semántica directa de las oraciones descriptivas. Esta es la tarea que nos ocupará en la siguiente sección.

\section{Una semántica de eventualidades}

Estamos ahora preparados para ocuparnos de nuestra tarea principal, a saber, el esbozo de una semántica de eventualidades adecuada para la representación de la predicación descriptiva. Hemos mencionado anteriormente que estamos interesados en la relaciones temporales entre eventualidades. En particular, daremos por hecho que dos eventualidades pueden estar relacionadas entre sí por medio de la inclusión temporal (Notación: $\sqsubseteq$ ) o de la intersección temporal (Notación: $\bigcirc$ ). De este modo, la fórmula e $\sqsubseteq$ e' expresa que la eventualidad e está incluida temporalmente dentro de e'. e $\bigcirc$ e', por otra parte, expresa que las eventualidades e y e' se intersectan temporalmente.

Otro de los presupuestos en los que nos basaremos es el siguiente. Los verbos y frases verbales corresponderán a predicados de eventualidades. Además, supondremos que las eventualidades asociadas con verbos desempeñan un papel central en la determinación del tipo de situación denotado por las oraciones que los contienen. De este modo, daremos por hecho la siguiente clasificación:

(11) a. arribar es un predicado de logro y por ende María arribó es una oración de logro,

b. hablar es un predicado de actividad y por ende María habló es una oración de actividad, 
c. escribir una carta es un predicado de realización y por ende María escribió una carta es una oración de resultado.

Antes de seguir adelante es importante recordar que deseamos tratar a la predicación descriptiva como la expresión de una relación entre dos eventualidades. Es crucial, para nosotros, determinar el tipo de eventualidad que corresponde al predicado secundario - típicamente un adjetivo. Una excursión bibliográfica nos muestra que existe un consenso general acerca de la interpretación de adjetivos. Vendler (1967, p. 108) adopta la idea de que los adjetivos son expresiones de estado.

Desde un punto de vista temporal, estar casado, estar presente o ausente, sano o enfermo, etc., se comportan como estados. Pero entonces podríamos dar un paso más y descubrir que esto es verdad para todas la cualidades. Ciertamente, algo es duro, caliente o amarillo durante un tiempo. Pero esto no significa que se estuviese dando, por ejemplo, un proceso de amarillamiento. De manera similar, aunque endurecer es un proceso (actividad o realización) estar duro es un estado.

Esta misma idea es defendida por Napoli (1989, p. 31) y por Higginbotham (1985). Según el primer autor:

Un adjetivo es siempre una expresión de eventualidad. Esto se debe a que los adjetivos denotan estados (ya sean estativos o activos).

En este artículo adoptaremos esta idea: los adjetivos son expresiones de eventualidad que denotan estados. Por eso daremos por hecho que la representación de las oraciones con predicación secundaria debe tener cuantificación sobre el estado denotado por el adjetivo y la eventualidad denotada por el predicado primario. 
Regresemos ahora a la representación de las oraciones en (1).

\subsection{Logros y predicación descriptiva}

Con respecto a (la), el punto de vista que estamos defendiendo supone un actor semántico, María, involucrado en dos eventualidades:

1. el estado que consiste en el estar contento de este actor, denotado por contento(e') $\wedge$ actor(e', María) y

2. el logro de la llegada de este actor, denotado por arribar $(\mathrm{e}) \wedge$ actor(e, María).

Estas consideraciones nos llevan por sí mismas a la siguiente representación provisional de (la):

(12) $\exists$ e e' $(\operatorname{arribar}(\mathrm{e}) \wedge$ actor $(\mathrm{e}$, María $) \wedge$ contenta(e') $\wedge$ actor(e', María)

Sin embargo, como hemos observado anteriormente, esta fórmula no es todo lo correcta que debiera ser. Ella no expresa de ninguna manera la relación temporal entre las dos eventualidades. Según nosotros, el uso de la inclusión temporal nos permite capturar este aspecto del significado de (la):

(13) $\exists$ e e'(arribar(e) $\wedge$ actor(e, María) $\wedge$ contento(e') $\wedge$ actor $\left(\mathrm{e}^{\prime}\right.$, María $\left.) \wedge \mathrm{e} \sqsubseteq \mathrm{e}^{\prime}\right)$

Esta segunda representación es satisfactoria dados los hechos que deseamos capturar. Nuestra oración original (la) es interpretada de modo que asevera la existencia de dos eventualidades que comparten un actor semántico y el evento que corresponde al predicado primario es visto como incluido temporalmente en la eventualidad correspondiente al predicado descriptivo. 


\subsection{Las actividades y la predicación descriptiva}

Las actividades son eventualidades que poseen la propiedad aditiva. Esto es, si e y e' son dos actividades temporalmente adyacentes, y ambas tienen la propiedad P, entonces la suma de estas actividades es una actividad con esta misma propiedad. Considérese la siguiente situación. Ayer, María habló durante dos horas. Durante la segunda hora María estuvo cansada. En esta situación (14b) puede suscitar inferencias erróneas:

$$
\begin{aligned}
& \text { a. María habló cansada } \\
& \text { b. ヨe e'e" (hablar(e) } \wedge \text { actor(e, María) } \wedge \text { cansa- } \\
& \text { da(e') } \wedge \text { actor }\left(e^{\prime}, \text { María }\right) \wedge \text { e } \sqsubseteq \text { e') }
\end{aligned}
$$

La cuestión es que (14b) sugiere que toda la actividad se desarrolló mientras María estuvo cansada. Hay, sin embargo, una fuerte tendencia a tomar toda la eventualidad y no solo una parte de ella para dar la interpretación a la variable.

Consideremos otro ejemplo. La oración

(15) María habló cansada y descansada

es consistente aunque su correspondiente representación no lo es:

$$
\begin{aligned}
& \exists \mathrm{e} \text { e'e" }(\text { hablar }(\mathrm{e}) \wedge \text { actor }(\mathrm{e}, \text { maría }) \wedge \text { cansado(e") } \\
& \wedge \text { actor(e", maría } \wedge \mathrm{e} \sqsubseteq \mathrm{e}^{\mathrm{e}} \wedge \operatorname{descansado}\left(\mathrm{e}^{\prime \prime}\right) \wedge \\
& \text { actor(e", maría) } \wedge \mathrm{e} \sqsubseteq \mathrm{e} ")
\end{aligned}
$$

Estas consideraciones nos llevan a concluir que la intersección temporal es una mejor elección para la representación de la relación temporal que deseamos establecer entre el predicado de actividad y el predicado descriptivo. Por esa razón nuestra propuesta usa la siguiente representación en la que la intersección toma el lugar de la inclusión: 


$$
\begin{aligned}
& \text { a. } \exists \text { e e'(hablar }(\mathrm{e}) \wedge \text { actor }(\mathrm{e}, \mathrm{a}) \wedge \text { dormido(e') } \wedge \text { ac- } \\
& \operatorname{tor}\left(e^{\prime}, \text { a) } \wedge \operatorname{actor}\left(e^{\prime}, \text { a }\right) \wedge e \bigcirc e^{\prime}\right) \\
& \text { b. } \exists \text { e e'e" (hablar(e) } \wedge \text { actor(e, maría) } \wedge \text { cansado(e') } \\
& \left.\wedge \text { actor }\left(e^{\prime}, \text { María }\right) \wedge \mathrm{e} \bigcirc \mathrm{e} ' \wedge \text { descansado(e" }\right) \wedge \\
& \operatorname{actor}\left(e^{\prime}, \text { María }\right) \wedge \text { e } \bigcirc \text { e") }
\end{aligned}
$$

\subsection{Realizaciones y predicación descriptiva}

A primera vista, la explicación dada para (la) podría generalizarse para explicar las oraciones de realización. La intersección es muy débil porque queremos en este caso que el estado descriptivo cubra toda la realización. La oración María escribió dos cartas nerviosa tiene para nosotros la interpretación que María se encuentra nerviosa al inicio de la eventualidad. Aun más, ella permanece en ese estado hasta el punto en que la eventualidad concluye. En primera instancia, pues, la fórmula en (18) captura el significado de (1c).

$$
\begin{aligned}
& \exists \mathrm{e} \mathrm{e}^{\prime}(\text { escribir-dos-cartas }(\mathrm{e}) \wedge \text { actor }(\mathrm{e}, \text { a }) \wedge \text { nervio- } \\
& \left.\mathrm{sa}^{\prime}\left(\mathrm{e}^{\prime}\right) \wedge \text { actor }\left(\mathrm{e}^{\prime}, \text { María }\right) \wedge \mathrm{e} \sqsubseteq \mathrm{e}^{\prime}\right)
\end{aligned}
$$

Veremos más adelante que con (18) no hemos dicho la última palabra. Pero por ahora esto será suficiente. Recapitulemos ahora brevemente para ver el punto en el que nos encontramos.

\subsection{Formulación final}

Para concluir esta sección revisemos lo que hemos hecho hasta ahora en la forma de condiciones informales para las representaciones semánticas. Así pues, una representación de una oración descriptiva debe contener

1. una eventualidad e que corresponde al predicado verbal. Por eso, el valor semántico de un verbo $\mathrm{V}$ deberá ser algo así como 
$\lambda \mathrm{v} \exists \mathrm{u}(\mathrm{V}(\mathrm{u}) \wedge \operatorname{actor}(\mathrm{u}, \mathrm{v}))$,

2. un estado e' que corresponde al predicado descriptivo. Por eso, el valor semántico del adjetivo A deberá ser algo así como

$\lambda \mathrm{v} \exists \mathrm{u}(\mathrm{A}(\mathrm{u}) \wedge \operatorname{actor}(\mathrm{u}, \mathrm{v}))$;

3. deberá haber un nivel, presumiblemente la frase verbal, en el que los actores de estas eventualidades sean identificados entre sí y una relación temporal sea aseverada entre las dos eventualidades:

$\lambda$ y $\lambda \mathrm{R} \exists \mathrm{z} \exists \mathrm{u}(\mathrm{V}(\mathrm{z}) \wedge \mathrm{A}(\mathrm{u}) \wedge \operatorname{actor}(\mathrm{z}, \mathrm{y}) \wedge \operatorname{actor}(\mathrm{u}$, y) $\wedge \mathrm{R}(\mathrm{z}, \mathrm{u}))$;

4. la combinación de este predicado complejo con su argumento puede ser reducida, vía reducción de lambda, a esta representación de la oración original;

$\lambda \mathrm{R} \exists \mathrm{e} \mathrm{e}^{\prime}\left(\mathrm{V}(\mathrm{e}) \wedge \mathrm{A}\left(\mathrm{e}^{\prime}\right) \wedge \operatorname{actor}(\mathrm{e}, \mathrm{a}) \wedge \operatorname{actor}\left(\mathrm{e}^{\prime}, \mathrm{a}\right) \wedge\right.$ $\left.\mathrm{R}\left(\mathrm{e}, \mathrm{e}^{\prime}\right)\right)$.

5. El resto del procedimiento se basa en una representación sintáctica que codifica información acerca del tipo de situación denotado por la oración. Esta información inducirá una representación que reemplaza la fórmula esquemática $R\left(e, e^{\prime}\right)$ por la relación temporal relevante:

(a) $\mathrm{S}($ LOGRO $)=$

i. $\lambda \mathrm{R} \exists \mathrm{e} \mathrm{e}^{\prime}\left(\mathrm{V}(\mathrm{e}) \wedge \mathrm{A}\left(\mathrm{e}^{\prime}\right) \wedge \operatorname{actor}(\mathrm{e}, \mathrm{a}) \wedge \mathrm{ac}-\right.$ $\left.\operatorname{tor}\left(\mathrm{e}^{\prime}, \mathrm{a}\right) \wedge \mathrm{R}\left(\mathrm{e}, \mathrm{e}^{\prime}\right)\right)(\sqsubseteq)$

$=$

ii. $\exists$ e $e^{\prime}\left(V(e) \wedge A\left(e^{\prime}\right) \wedge\right.$ actor $(e, a) \wedge$ actor $\left(e^{\prime}, a\right)$ $\left.\wedge \sqsubseteq\left(\mathrm{e}, \mathrm{e}^{\prime}\right)\right)$

(b) $\mathrm{S}($ ACTIDAD) $=$

i. $\lambda \mathrm{R} \exists \mathrm{e} \mathrm{e}^{\prime}\left(\mathrm{V}(\mathrm{e}) \wedge \mathrm{A}\left(\mathrm{e}^{\prime}\right) \wedge \operatorname{actor}(\mathrm{e}, \mathrm{a}) \wedge \mathrm{ac}-\right.$ $\left.\operatorname{tor}\left(e^{\prime}, a\right) \wedge R\left(e, e^{\prime}\right)\right)(\bigcirc)$

$=$ 


$$
\begin{aligned}
& \text { ii. } \exists \mathrm{e} \mathrm{e}^{\prime}\left(\mathrm{V}(\mathrm{e}) \wedge \mathrm{A}\left(\mathrm{e}^{\prime}\right) \wedge \operatorname{actor}(\mathrm{e}, \mathrm{a}) \wedge \operatorname{actor}\left(\mathrm{e}^{\prime}, \mathrm{a}\right)\right. \\
&\left.\wedge \bigcirc\left(\mathrm{e}, \mathrm{e}^{\prime}\right)\right) \\
& \text { (c) } \mathrm{S}(\mathrm{REALIZACIÓN})= \\
& \text { i. } \lambda \mathrm{R} \exists \mathrm{e} \mathrm{e}^{\prime}\left(\mathrm{V}(\mathrm{e}) \wedge \mathrm{A}\left(\mathrm{e}^{\prime}\right) \wedge \operatorname{actor}(\mathrm{e}, \mathrm{a}) \wedge \mathrm{ac}-\right. \\
& \\
&\left.\operatorname{tor}\left(\mathrm{e}^{\prime}, \mathrm{a}\right) \wedge \mathrm{R}\left(\mathrm{e}, \mathrm{e}^{\prime}\right)\right)(\sqsubseteq) \\
&= \\
& \text { ii. } \exists \mathrm{e} \mathrm{e}^{\prime}\left(\mathrm{V}(\mathrm{e}) \wedge \mathrm{A}\left(\mathrm{e}^{\prime}\right) \wedge \operatorname{actor}(\mathrm{e}, \mathrm{a}) \wedge \operatorname{actor}\left(\mathrm{e}^{\prime}, \mathrm{a}\right)\right. \\
&\left.\wedge \sqsubseteq\left(\mathrm{e}, \mathrm{e}^{\prime}\right)\right)
\end{aligned}
$$

Desde la perspectiva adoptada en este artículo, esto es lo más que deseamos obtener. El paso siguiente es, naturalmente, el desarrollo de un marco sintáctico que incorpore los puntos previos. Esto es algo que no vamos a considerar aquí. En lugar de eso, tornamos nuestra atención hacia la relación de la representación propuesta y las paráfrasis basadas en la conectiva temporal 'cuando'.

\section{Oraciones temporales y predicación descriptiva}

En esta sección argumentaremos que el análisis que Dowty hizo de la predicación descriptiva está en armonía con la propuesta que hemos ofrecido hasta aquí. Nuestra aseveración es que las oraciones de tipo cuando inducen una relación temporal que coincide con las relaciones presentadas anteriormente.

\subsection{Heinämäki sobre When}

En su disertación Orvokki Heinämäki ofrece un análisis de las oraciones formadas con la conectiva temporal when (Heinämäki 1974, pp. 24-28). En nuestra exposición partimos de la idea de que las oraciones se corresponden con intervalos. Sea $\mathrm{B}$ una oración durativa. Una oración de la forma $A$ cuando $B$ significa 
- Si A es logro entonces el intervalo A es un subintervalo del intervalo B.

- Si A es durativa entonces A y B se intersectan.

- Si A es una realización entonces el intervalo A es un subintervalo del intervalo B.

Para determinar la relación entre nuestra semántica y el análisis de Heinämäki sólo necesitamos hacer una observación terminológica. La expresión oración durativa denota actividades o estados. Por ende, dejando a B como una oración de estado vemos que los predicados descriptivos y las oraciones de tipo cuando inducen la misma relación temporal. Un corolario a esta observación es que nuestra semántica para la predicación descriptiva y la semántica de Dowty coinciden, suponiendo que le damos a la conectiva temporal el mismo análisis que propone Heinämäki.

Naturalmente, el análisis de Heinämäki es mucho más general y detallado, pero nosotros solamente estamos interesados en los casos en los que el predicado descriptivo y la cláusula temporal subordinada pertenecen al mismo tipo de situación. En las siguientes secciones comentaremos algunos de los puntos que han llevado al rechazo de las ideas de Dowty. El objetivo de nuestra observación es demostrar lo siguiente: La existencia de dos paráfrasis sintácticamente diferentes no tiene contenido semántico. Ambas paráfrasis cubren la misma área semántica.

\subsection{La crítica a Dowty}

Como se mencionó, (19a) tiene las interpretaciones (19b) y (19c):

(19) a. María se casó joven

b. María se casó cuando estaba joven

c. Cuando María se casó ella estaba joven 
De una manera u otra, esta multiplicidad de interpretaciones ha contribuido a socavar la aceptabilidad de la propuesta de Dowty. En este artículo argüimos que, afino la semántica de Heinämäki, estas dos interpretaciones son equivalentes. El argumento es éste: Según Heinämäki, (19c) expresa intersección mientras que (19b) expresa inclusión. Pero en lo que respecta a los logros, como éstos son conceptualizados como eventualidades puntuales, intersección e inclusión coinciden. (La analogía con los conjuntos unitarios debe hacer las cosas transparentes: en ellos coinciden intersección e inclusión.)

Por lo tanto, como Dowty mismo notó, la existencia de dos interpretaciones temporales no cuenta en contra de su propuesta porque éstas resultan equivalentes.

De hecho, nosotros podemos elaborar este resultado. No se trata simplemente que las dos interpretaciones temporales de (19a) resulten ser equivalentes. Se trata en realidad de que todos los casos de multiplicidad interpretativa que se pueden dar admiten el mismo tipo de explicación. Dentro del marco de la semántica temporal de Heinämäki la siguiente proposición es válida:

(20) Si B es una oración de estado, entonces $A$ cuando $B$ y $B$ cuando $A$ son equivalentes.

Para convencernos de que esta proposición es válida, debemos expandir nuestro comentario sobre Heinämäki. Según ella,

- Si A es un logro y B es durativa, entonces $B$ cuando $A$ significa que el intervalo A y el intervalo $\mathrm{B}$ se intersectan.

- Si A es una realización y $\mathrm{B}$ es durativa, entonces $B$ cuando $A$ significa que el intervalo A está incluido en el intervalo B.

Esta aseveración es demostrada por casos. 
A es una oración de actividad y $B$ es una oración de estado. La intersección del intervalo A y el intervalo B corresponde a $A$ cuando $B$ y $B$ cuando $A$.

A es una oración de logro y $B$ es una oración de estado. En este caso, $A$ cuando $B$ corresponde a la inclusión de A en B. $B$ cuando $A$, por otra parte, corresponde a la intersección. Pero ya hemos visto que estas dos relaciones coinciden con respecto a los logros.

A es una oración de realización y $B$ es una oración de estado. En este caso $A$ cuando $B$ y $B$ cuando $A$ corresponden ambas a la inclusión de A en B. Q.E.D.

La proposición demuestra que la cuestión referente a la paráfrasis apropiada para oraciones descriptivas no es más que un problema aparente. No existe ninguna discrepancia semántica estre estas dos alternativas — suponiendo que los predicados descriptivos corresponden a estados.

\subsection{Heinämäki y Mientras}

Hemos mencionado que algunos lingüistas prefieren una paráfrasis basada en la conectiva temporal mientras en lugar de la interpretación basada en cuando. Esta preferencia induce igualmente el rechazo del análisis que debemos a Dowty. Lo que argumentaré aquí es que, semánticamente, no hay modo alguno de diferenciar entre estas interpretaciones. En la teoría de Heinämäki una oración del tipo $A$ mientras $B$ está bien formada siempre y cuando $\mathrm{B}$ no corresponda a un logro. Por consiguiente, el caso en que B corresponde a un estado es un caso especial. Las relaciones temporales inducidas por esta conectiva son familiares para nosotros:

1. Si A es una actividad o logro, entonces $A$ mientras $B$ significa que el intervalo A y el intervalo B se intersectan. 
2. Si A es una realización, entonces $A$ mientras $B$ significa que el intervalo $A$ esta temporalmente incluido en el intervalo B.

A partir de esto es claro, pues, que las relaciones temporales inducidas por las paráfrasis basadas en cuando y mientras coinciden dentro del marco ofrecido por la semántica de Heinämäki. Una indicación convincente de que la base sobre la cual el análisis de Dowty ha sido rechazado, carece de firmeza.

\section{Ajustando la semántica}

La semántica que hemos descrito aquí es sólo una aproximación inicial. En esta sección discutimos algunos casos que demandan una semántica un poco más compleja.

\subsection{Predicación descriptiva orientada hacia el objecto}

En primer lugar, nosotros hemos restringido nuestra atención a la predicación descriptiva orientada hacia el sujeto. Pero, por supuesto, existen también predicados secundarios que se orientan al objeto. De hecho, la teoría de Dowty fue inspirada por la propuesta inicialmente formulada en Green (1970). El interés de Georgia Green se centró en los predicados descriptivos que son orientados al objecto y no al subjecto. La oración siguiente ilustra el caso que le concierne:

(21) Ella toma el café caliente

Más aún, la paráfrasis basada en la conectiva cuando formaba ya parte de su análisis de la predicación descriptiva. Según ella, el contenido de (21) sería capturado por (22):

(22) Ella toma café cuando éste está caliente

La existencia de la predicación descriptiva que está orientada al objeto fue usada en Nichols (1978) para cuestionar la 
paráfrasis basada en cuando. Consideremos por un instante la oración que está aquí abajo y su supuesta interpretación:

(23) a. Quemaron viva a María

b. $\exists$ e e' $($ quemar $(e) \wedge$ Objecto(e, a) $\wedge$ vivo(e') $\wedge$ Agente $\left(\mathrm{e}^{\prime}\right.$, a) $\left.\wedge \mathrm{e} \sqsubseteq \mathrm{e}^{\prime}\right)$

La interpretación codificada por (23b) resulta insatisfactoria. Esta fórmula nos invita a inferir que el proceso de quemar tuvo lugar mientras el objeto estaba vivo. El problema en realidad es el siguiente: supongamos que el quemar al objeto culmina en su muerte pero que este proceso continúa más allá del momento de expiración. En este caso 23 a sería verdadera pero 23 b no.

La solución a este problema es la siguiente. Las eventualidades pueden ser factorizadas en fases:

1. fase inicial

2. momento de culminación

3. fase final

Según nosotros, 23a expresa que la fase inicial y el punto de culminación del proceso de quemar están ambos incluidos en el estado de vivir del objeto. De este modo, todo lo que tenemos que hacer es expandir el lenguaje con nuevos predicados de eventualidades. Para toda eventualidad e, FI(e), CP(e) y FP(e) denotarán, respectivamente, la fase inicial de e, su momento de culminación, o su fase final. Estos predicados son usados en la representación de 23a que evita los problemas de $23 \mathrm{~b}$

(24) $\exists$ e e'(quemar(e) $\wedge$ Objeto(e, a) $\wedge$ vivo(e') $\wedge$ actor $\left(e^{\prime}\right.$, a) $\left.\wedge \mathrm{IP}(\mathrm{e}) \sqsubseteq \mathrm{e}^{\prime} \wedge \mathrm{CP}(\mathrm{e}) \sqsubseteq \mathrm{e}^{\prime}\right)$ 


\subsection{Otro uso de la factorización}

La semántica que hemos desarrollado en las secciones precedentes ignora todas las cuestiones relacionadas con la distinción aspectual entre eventualidades perfectivas e imperfectivas. Las eventualidades que hemos estado considerando ejemplifican todas casos perfectivos, en el sentido de que las eventualidades son consideradas como completas - acabadas. Pero en los lenguajes naturales es posible distinguir entre oraciones imperfectivas y oraciones perfectivas. Siendo esto así, una cuestión natural es la de preguntarse de que manera afecta esto la representación de oraciones que involucran el punto de vista imperfectivo y predicación secundaria. Considérese, por ejemplo, la versión imperfectiva de la oración perfectiva (la):

(25) María llegaba contenta

La interpretación de esta oración no está en armonía con la semántica discutida en este artículo. La cuestión es que en su interpretación estándar esta oración significa que la eventualidad de llegar, de la cual María es el actor, no necesita haber alcanzado su punto de culminación en el momento en que nosotros procedemos a evaluarla. La conclusión que extraemos de aquí es que una condición necesaria para la representación adecuada de las oraciones descriptivas imperfectivas requiere el empleo de la factorización que hemos presentado. En este caso podemos representar la interpretación descriptiva de la manera siguiente:

(26) $\exists$ e e'(llegar(e) $\wedge$ cansado(e') $\wedge$ actor(e, María $) \wedge$ actor $\left(\mathrm{e}^{\prime}\right.$, María $\left.) \wedge \mathrm{IP}(\mathrm{e}) \sqsubseteq \mathrm{e}^{\prime}\right)$

\section{Restricciones sobre los predicados descriptivos}

Hasta ahora no hemos dicho nada acerca del tipo de adjetivos que pueden ser usados descriptivamente. Sin embargo, 
no todos los adjetivos son igualmente satisfactorios en este papel. Los hablantes del español pueden notar que nuestra explicación pasa por alto la distinción entre predicados de tipo ser y predicados de tipo estar. Está distinción desempeña, aparentemente, un papel primordial en los juicios de aceptación gramatical. Las oraciones siguientes parecen, por ejemplo, menos aceptables que aquéllas en (1)

(27) a. María llegó socialista

b. María protestó holandesa

c. María escribió la carta zarca

Existen como mínimo tres explicaciones para la baja aceptabilidad de estas oraciones

1. En primer lugar los adjetivos socialista, zarca, holandesa son combinados normalmente con estar en oraciones copulativas. La generalización es entonces:

Sólo los adjetivos combinables con estar son predicados descriptivos.

2. Los adjetivos usados en estas oraciones expresan propiedades de individuos que, en las palabras de Dowty, "no pueden ser perdidas o adquiridas con el paso del tiempo". La generalización sería entonces

Sólo los predicados restringidos temporalmente son predicados descriptivos.

3. De acuerdo con la distinción presentada en Carlson (1977), uno distingue dos tipos de predicados: Los predicados de fase corresponden a propiedades transitorias de individuos mientras que los predicados de nivel individual corresponden a propiedades más o menos estables. Según esta terminología los adjetivos 
en nuestras oraciones serían predicados de nivel individual. La generalización, sugerida en Grubig (1992) es la siguiente:

Sólo los predicados de fase son predicados descriptivos.

No está del todo claro si estas generalizaciones son válidas o no. El problema es que es muy difícil encontrar adjetivos que resistan una interpretación temporal. Por ejemplo, la siguiente oración que contiene predicados de tipo ser es aceptable:

(28) María llegó socialista y se fue apolítica

Incidentalmente, la sugerencia de que (28) es aceptable porque señala un cambio de estado está abierta a crítica. El cambio de estado no es de ninguna forma necesario. Por ejemplo, en la oración siguiente el cambio está ausente:

(29) María llegó socialista y se fue socialista

Quizá podamos expresar estas cosas en la forma siguiente. Si los predicados que son normalmente predicados de nivel individual son usados en predicación secundaria, entonces su contribución al significado de las oraciones en las que aparecen es similar a la contribución de los predicados de fase. Estos denotan manifestaciones espacio-temporales de sus argumentos. O, como Dowty mismo se inclina a explicar la situación, la inaceptabilidad de nuestras oraciones debe ser explicada en términos de nuestras creencias sobre el mundo real y no en términos de la gramática. $\mathrm{Si}$ somos capaces de modificar nuestros juicios acerca de la realidad extra lingüística, esto afectará nuestros juicios de aceptabilidad gramátical. El uso típico de los adjetivos que se combinan con ser es extraño al uso descriptivo. Pero si modificamos nuestra visión de la realidad entonces estos podrán funcionar de esta manera. 


\section{Una representación alternativa}

Ahora hemos llegado al final de nuestra exposición. Antes de concluir es importante relacionar nuestra semántica con una representación equivalente a ella. Supongamos que identificamos un logro con su momento de culminación. En este caso obtenemos la siguiente representación de (la)

(30) $\exists$ e e' $\left(\operatorname{arribar}(\mathrm{e}) \wedge\right.$ actor $\left(\mathrm{e}\right.$, a) $\wedge$ contenta(e') $\wedge$ actor $\left(\mathrm{e}^{\prime}\right.$, a) $\left.\wedge \mathrm{CP}(\mathrm{e}) \bigcirc \mathrm{e}^{\prime}\right)$

Pongámosle antes de finalizar un poco de atención a un modo alternativo de representar el contenido de (30). Póngase $\mathrm{CP}(\mathrm{e}, \mathrm{t})$ con el significado de que la eventualidad e culmina en el momento $t$, mientras que $\mathrm{H}(\mathrm{e}, \mathrm{t})$ significa que el estado e vale en el momento t. Entonces la siguiente fórmula tiene el mismo contenido que la fórmula previa:

(31) $\exists$ e e't $\left(\operatorname{arribar}(\mathrm{e}) \wedge\right.$ actor $(\mathrm{e}, \mathrm{a}) \wedge \operatorname{contenta}\left(\mathrm{e}^{\prime}\right) \wedge$ actor $\left(\mathrm{e}^{\prime}\right.$, a) $\wedge \mathrm{CP}(\mathrm{e}, \mathrm{t}) \wedge \mathrm{H}\left(\mathrm{e}^{\prime}, \mathrm{t}\right)$

Esta fórmula expresa la existencia de las eventualidades de arribar y de estar contento. Más aún, expresa que estas eventualidades comparten un actor y un momento. Pero éste es sólo un modo diferente de expresar el contenido de nuestra representación de (la). Lo que debemos tener en cuenta aquí es que esta última fórmula es la representación que Larson y Segal ofrecen para las oraciones de logro con predicados descriptivos. No hay ninguna razón para suponer que las oraciones de realización y de actividad no puedan ser representadas de esta misma manera.

\section{Palabras finales}

En este artículo hemos intentado desarrollar una representacion semántica de las oraciones que expresan predicación secundaria. Nuestro análisis semántico vincula la semántica de esta construcción con la semántica de los tipos de 
acción. Esto nos ha permitido aclarar un malentendido que se ha dado en torno a la interpretación de las oraciones con predicación secundaria.

\section{BIBLIOGRAFÍA}

Bach, Emmon, 1981, "On Time, Tense and Aspect: An Essay on English Metaphysics", Radical Pragmatics, Peter Cole (ed.), Academic Press, , pp. 62-81.

Carlson, Gregory, 1977, Reference to Kinds in English, Doctoral dissertation, University of Massachusetts.

Demonte, Violeta, 1988, "Remarks on Secondary Predicates: C-command, extraction, and Reanalysis", The Linguistic Review, 6, 1-39.

Dowty, David, 1972, "Temporally Restricted Predicates", Suntax and Semantics, John P. Kimball (ed.), Academic Press, pp. 51-62.

Green, Georgia M., 1970, "How Abstract is Surface Structure?", Papers from the Sixth Regional Meeting, CSLI, R.I. Binnick et al. (ed.), University of Chicago, pp. 270-281.

Grubig, H. Bernhard, 1992, "Zur Frage der grammatischen Repräsentation thetischer und kategorischer Sätze", Informatiosstruktur und Grammatik, Joachim Jacobs (ed.), Linguitische Berichte, Sonderheft, no. 4, Westdeutscher Verlag.

Guemann, Steven Allen, 1990, Secondary Predication in English and Spanish, Doctoral dissertation, University of California, Berkeley.

Heinämäki, Orvokki, 1974, Semantics of English Temporal Connectives, Doctoral dissertation, University of Helsinki. Reproduced by Indiana University Linguistics Club.

Higginbotham, James, 1985, "On Semantics", Linguistic Inquiry, 16(4), pp. 547-592.

Larson, Richard and Gabriel Segal, 1995, Knowledge and Meaning. An Introduction to Semantic Theory, The MIT Press.

Luján, Marta, 1980, Sintaxis y semántica del adjetivo, Ediciones Cátedra.

Napoli, Donna Jo., 1989, Predication Theory, Cambridge University Press. 
Nichols, Johanna, 1978, "Secondary Predicates", Proceedings of the Fourth Annual Meeting of the Berkeley Linguistic Society, Berkeley Linguistic Society, pp. 114-127.

Parsons, T., 1985, "Underlying Events in the Logical Analysis of English", Actions and Events: Perspectives on the Philosophy of Donald Davidson, E. LePore and B. McLaughlin (eds.), Basil Blackwell, pp. 235-267.

Real Academia de la Lengua (ed.), 1985, Esbozo de una nueva gramática de la lengua española, Espasa-Calpe.

Rothstein, Susan D., 1985, The Syntactic Forms of Predication, Doctoral dissertation, Bar-llan University.

Vendler, Zeno, 1967, Linguistics in Philosophy, Chap. Verbs and Times, Cornell University Press.

Winkler, Susanne, 1996, Focus and Secondary Predication, Mouton de Gruyter.

Recibido: 30 de septiembre de 1999 


\section{SUMMARY}

In this paper we outline the semantics of the class of sentences represented below:

María llegó contenta

María arrived happy

María habló dormida

María spoke in her dreams

María escribió la carta nerviosa

María wrote the letter nervous

The term depictive is generally used to refer to the secondary predicate that occur in these sentences - the adjectives contenta, dormida, nerviosa - in agreement with the subject term. We adopt this terminology and in this papers we shall speak of depictive predicates, predication and sentences. We assume, uncontroversially, that each depictive sentence expresses a temporal relation between its primary predicate (the verbal predicate) and its secondary predicate (the adjectival predicate). We then show that this temporal relation is closely related to the formal semantics of temporal when-clauses.

The theoretical background for our treatment is found in the works of Donald Davidson and David Dowty. We shall rehearse the most basic features of Davidson's treatment of adverbial modification. After that we present Dowty's view on depictive predication. The central task of our presentation is to unify the two previous themes. Roughly speaking, the proposal we develop in this lecture casts Dowty's basic intuition within an event based semantics.

We also discuss the role of the Spanish imperfective past in the understanding of depictive sentences and its consequences for our semantics. In addition to that, we address the question of the low acceptability of the so-called ser adjectives in depictive sentences. We point out that this question is related to the (cross-linguistically attested) low acceptability of individual-level predicates in such sentences. 